\title{
On Quadratic First Integrals of the Geodesic Equations for Type $\{22\}$ Spacetimes
}

\author{
Martin Walker and Roger Penrose \\ Department of Mathematics, Birkbeck College, London, England
}

Received May 1, 1970

\begin{abstract}
It is shown that every type $\{22\}$ vacuum solution of Einstein's equations admits a quadratic first integral of the null geodesic equations (conformal Killing tensor of valence 2), which is independent of the metric and of any Killing vectors arising from symmetries. In particular, the charged Kerr solution (with or without cosmological constant) is shown to admit a Killing tensor of valence 2 . The Killing tensor, together with the metric and the two Killing vectors, provides a method of explicitly integrating the geodesics of the (charged) Kerr solution, thus shedding some light on a result due to Carter.
\end{abstract}

In a remarkable paper, Carter [1] has shown how to integrate the geodesic equations in a class of solutions of Einstein's equations including the (charged) Kerr solution [2], thus reducing the geodesic problem to one of quadratures. Carter's method depends on a peculiar feature of these solutions, namely that "the Hamilton-Jacobi equation (for the geodesic problem) can be solved by separation of variables", in a particular coordinate system [3]. In the present work we obtain an alternative procedure which achieves the same ends, but which is in some respects more transparent than Carter's. In addition, our method provides an explicit integration of all null geodesics in any type $\{22\}$ vacuum solution. As yet, however, we have not been able to adapt our method so as to obtain charged particle orbits.

By a (vacuum) solution we understand a pair $\left(M, g_{a b}\right)$ consisting of a 4-dimensional connected Hausdorff differentiable manifold $M$, and a pseudo-Riemannian metric $g_{a b}$ of signature (+- -) defined on $M$ satisfying Einstein's (vacuum) gravitational field equations (with or without cosmological constant). In order to interpret the physical significance of a vacuum) solution, it is frequently useful to understand the global nature ${ }^{1}$ of the spacetime $\left(M, g_{a b}\right)$. The global analysis of a (vacuum) solution, however, is usually a difficult task since in most cases $g_{a b}$ is given only locally. A knowledge of four first integrals of the geodesic

${ }^{1}$ For example, the nature of singularities [4] and conformal infinity [5]. 
equations $t^{c} \nabla_{c} t^{a}=0$ greatly facilitates the extension of a locally given spacetime to a maximal inextendible spacetime (and hence the global analysis of $\left.\left(M, g_{a b}\right)\right)$, since in this case an explicit construction can, in principle, be carried out [6]. Theorems which assert the existence of first integrals are, therefore, of interest in general relativity.

The spacetime $\left(M, g_{a b}\right)$ is said to admit a first integral of degree $m$ if a scalar $\chi$, satisfying $t^{c} \nabla_{c} \chi=0$, is defined along any geodesic with parallely propagated tangent vector $t^{a}$, where $\chi$ is of the form $\chi=K_{a_{1} \ldots a_{m}} t^{a_{1}} \ldots t^{a_{m}}$ for some tensor $K_{a_{1} \ldots a_{m}}$. The requirement that $\chi$ be constant along geodesics is equivalent to the equation ${ }^{2}$

$$
\nabla_{(a} K_{\left.a_{1} \ldots a_{m}\right)}=0 .
$$

We shall call a (totally symmetric) solution of Eq. (1) a Killing tensor of valence $m$. When $m=2$, we shall refer to $\chi$ and $K_{a_{1} \ldots a_{m}}$ as quadratic.

The case $m=1$ is well known, and Eq. (1) is then Killing's equation $\nabla_{(a} \xi_{b)}=0$. Moreover, the vector $\xi^{a}$ has a direct geometrical interpretation as the tangent to the orbit of a point of $M$ under the action of a oneparameter group of isometries of $\left(M, g_{a b}\right): \mathscr{L}_{\xi} g_{a b}=0$. No such direct geometrical interpretation of $K_{a_{1} \ldots a_{m}}$ for $m>1$ satisfying Eq. (1) appears to be known.

In certain cases of global analysis, it is sufficient to know the conformal structure of $\left(M, g_{a b}\right)$, for example, in the study of conformal infinity [5]. In this case, a knowledge of four first integrals of the null geodesics will suffice. The spacetime $\left(M, g_{a b}\right)$ is said to admit a conformal Killing tensor of valence $m$ if there exist on $M$ tensors $K_{a_{1} \ldots a_{m}}$ and $k_{a_{1} \ldots a_{m-1}}$ such that

$$
\nabla_{(a} K_{\left.a_{1} \ldots a_{m}\right)}=k_{\left(a a_{1} \ldots a_{m-2}\right.} g_{\left.a_{m-1} a_{m}\right)} .
$$

Eq. (2) is equivalent to the requirement that $K_{a_{1} \ldots a_{m}} l^{a_{1}} \ldots l^{a_{m}}$ be constant along null geodesics with parallely propagated tangent vector $l^{a}$. In particular, for $m=1$ we have $\nabla_{a} \xi_{b)}=\varphi g_{a b}$ for some scalar $\varphi$, and the conformal Killing vector $\xi^{a}$ defines a conformal symmetry of $\left(M, g_{a b}\right)$ in much the same way that a Killing vector defines an isometry of $\left(M, g_{a b}\right)$. Again however, no such interpretation of $K_{a_{1} \ldots a_{m}}$ for $m>1$ satisfying Eq. (2) appears to be known.

It will be observed that any fixed linear combination of symmetrized outer products of (conformal) Killing tensors is again a (conformal) Killing tensor, and that the metric itself is a Killing tensor. A (conformal) Killing tensor which is constructible from the metric and other (conformal) Killing tensors in this way will be called reducible, otherwise irreducible.

${ }^{2}$ Round brackets denote symmetrization of the indices enclosed; square brackets denote antisymmetrization. 
In this paper we investigate the case $m=2$, the existence of quadratic (conformal) Killing tensors, for vacuum solutions whose Weyl tensor ${ }^{3}$ is of type $\{22\}$. We will prove the following:

Theorem 1. Every type $\{22\}$ vacuum solution (with or without cosmological constant) admits a quadratic conformal Killing tensor which is irreducible provided the spacetime admits fewer than four independent Killing vectors.

Since every type $\{22\}$ vacuum solution admits either two or four independent Killing vectors, it is a consequence of Theorem 1 that four first integrals of the null geodesics equations for these solutions can be written down explicitly. More particularly, we also prove

Theorem 2. The charged Kerr solution with or without cosmological constant $[1,2,10]$ admits a quadratic Killing tensor which is irreducible provided the angular momentum parameter a is not zero.

The proofs consist of explicitly constructing the tensor whose existence is asserted. The construction follows from the spinor form of the second Bianchi identity.

Let $C_{a b c d}$ be the Weyl tensor of a vacuum solution. Then since $R_{a b}=R_{a c b}^{c}=\Lambda g_{a b}$, where $R_{a b c d}$ is the Riemann tensor and $\Lambda$ the cosmological constant, the second Bianchi identity $\nabla_{[a} R_{b c] d e}=0$ becomes $\nabla^{a} C_{a b c d}=0$. The Weyl spinor $\Psi_{A B C D}=\Psi_{(A B C D)}$ is defined by ${ }^{4}$

$$
C_{a b c d}=\Psi_{A B C D^{\prime}} \varepsilon_{A^{\prime} B^{\prime}} \varepsilon_{C^{\prime} D^{\prime}}+\varepsilon_{A B} \varepsilon_{C D} \bar{\Psi}_{A^{\prime} B^{\prime} C^{\prime} D^{\prime}}
$$

so the spinor Bianchi identities are

$$
\nabla^{A A^{\prime}} \Psi_{A B C D}=0
$$

where $\varepsilon_{A B}=-\varepsilon_{B A}, \varepsilon_{A}{ }^{A}=2$, and $\nabla_{c} \varepsilon_{A B}=0$ with $\varepsilon_{A B} \varepsilon_{A^{\prime} B^{\prime}}=g_{a b}$. Let $o^{A}, l^{A}$ be a spinor dyad satisfying $o_{A} l^{A}=1$. Then the real null vectors $l^{a}=o^{A} \bar{o}^{A^{\prime}}$ and $n^{a}=l^{A} l^{A^{\prime}}$ together with the complex null vectors $m^{a}=o^{A} l^{A^{\prime}}$ and $\bar{m}^{a}=l^{A} \bar{o}^{A^{\prime}}$ form a null tetrad $\left\{l^{a}, n^{a}, m^{a}, \bar{m}^{a}\right\}$ on $M[12]$.

The Weyl spinor is completely determined by the five complex scalars $\Psi_{0}, \ldots, \Psi_{4}$ of Newman and Penrose [12] defined in terms of $\Psi_{A B C D}$ and the spinor dyad $o^{A}, l^{A}$. In the case of a type $\{22\}$ Weyl spinor,

${ }^{3}$ See, for example, Pirani [9], Penrose [11].

${ }^{4}$ We use "Batelle conventions" for tensors and spinors: see Penrose [11]. Accordingly, latin indices are just abstract labels which do not take on numerical values; hence, they do not refer to any coordinate system or tetrad basis. On the other hand, Gothic indices do refer to tensor or spinor components in the normal way. These conventions allow one to write the tensor-spinor correspondence without " $\sigma$ 's", e.g., $g_{a b}=\varepsilon_{A B} \varepsilon_{A^{\prime} B^{\prime}}, \delta_{a}^{b}=\varepsilon_{A}{ }^{B} \varepsilon_{A^{\prime}}{ }^{B^{\prime}}$, $g^{a b}=\varepsilon^{A B} \varepsilon^{A^{\prime} B^{\prime}}$. The spinor "Kronecker delta" is written $\varepsilon_{A}{ }^{B}$ to avoid possible confusion in raising and lowering its spinor indices. 
$\Psi_{0}=\Psi_{1}=\Psi_{3}=\Psi_{4}=0$ provided $o^{A}$ and $l^{A}$ are chosen to be repeated principal spinors of $\Psi_{A B C D}$ (so that the corresponding null vectors $l^{a}$ and $n^{a}$ lie along the repeated principal null directions of $C_{a b c d}$ ). The only freedom, apart from $o^{A} \rightarrow l^{A}, l^{A} \rightarrow-o^{A}$, in the choice of $o^{A}$ and $l^{A}$ for a type $\{22\}$ vacuum solution is the two parameter connected subgroup of the Lorentz group acting in the tangent space at each point which leaves the corresponding null directions invariant. The action of this subgroup on $o^{A}, l^{A}$ is given by $o^{A} \rightarrow \lambda o^{A}, l^{A} \rightarrow \lambda^{-1} l^{A}$ where $\lambda$ is a complex scalar. The normalization $o_{A} l^{A}=1$ is preserved.

If we let $\psi=6 \Psi_{2}$, then the Weyl spinor of a type $\{22\}$ vacuum solution is given by

$$
\Psi_{A B C D}=\psi o_{(A} o_{B} l_{C} l_{D)} \text {. }
$$

We proceed to prove Theorems 1 and 2 by a sequence of lemmas.

Lemma 1. Let $\chi_{B C}=\psi^{-1 / 3} o_{(B} l_{C)}$. Then

$$
\nabla_{\left(A^{\chi}{ }_{B C}\right.}^{A^{\prime}}=0 \text {. }
$$

Proof. Consider the quantity $\nabla_{(A}^{A^{\prime}}\left[\psi^{n} o_{B} l_{C)}\right]$. We shall show that, if $n=-1 / 3$, every dyad component of this quantity vanishes by virtue of the vanishing of the corresponding component of Eq. (3).

Writing out the Bianchi identity (3) using equation (4) and transvecting with $\psi^{-1} \imath^{B} O^{C} O^{D}$ gives

$$
\begin{aligned}
-\frac{1}{3} \psi^{-1} o_{A} \nabla^{A A^{\prime}} \psi-\frac{1}{3} \nabla^{A A^{\prime}} o_{A}+l^{B} O^{C} o^{D} \\
\cdot\left[l_{A} \nabla^{A A^{\prime}}\left(l_{(B} o_{C} o_{D}\right)+o_{A} \nabla^{A A^{\prime}}\left(l_{(B} l_{C} o_{D}\right)\right]=0 .
\end{aligned}
$$

Expanding out the terms in square brackets and making use of the relation $\varepsilon_{A}{ }^{D}=\imath^{D} o_{A}-o^{D} l_{A}$, we find

$$
\psi^{-1} o_{A} \nabla^{A A^{\prime}} \psi-3 o^{D} l_{A} \nabla^{A A^{\prime}} o_{D}=0 .
$$

The analogous component of $\nabla_{(A}^{A^{\prime}}\left[\psi^{n} o_{B} l_{C}\right]$ is

$$
o^{A} o^{B} l^{C} \nabla_{(A}^{A^{\prime}}\left[\psi^{n} o_{B} l_{C}\right]=\frac{1}{3} n \psi^{n}\left[\psi^{-1} o_{A} \nabla^{A A^{\prime}} \psi+\frac{1}{n} o^{D} l_{A} \nabla^{A A^{\prime}} o_{D}\right]
$$

which vanishes, by Eq. (6), if $n=-1 / 3$. Therefore

and similarly

$$
o^{A} o^{B} l^{C} \nabla_{(A}^{A^{\prime}}\left[\psi^{-1 / 3} o_{B} l_{C}\right]=0,
$$

$$
o^{A} \imath^{B} l^{C} \nabla_{(A}^{A^{\prime}}\left[\psi^{-1 / 3} o_{B} l_{C}\right]=0 .
$$

The components $o^{A} o^{B} o^{C} \nabla_{(A}^{A^{\prime}}\left[\psi^{-1 / 3} o_{B} l_{C}\right]$ and $\left.\imath^{A} l^{B} l^{C} \nabla_{(A}^{A^{\prime}}\left[\psi^{-1 / 3} o_{B} l_{C}\right)\right]$ vanish by virtue of the shear-free conditions on $o^{A}$ and $l^{A}$, which again are consequences of (3). Hence $\nabla_{(A}^{A^{\prime}}\left[\psi^{-1 / 3} o_{B} l_{C)}\right]=0$. 
Before completing the proof of Theorem 1 we need to translate Eq. (5) into a tensor form. To this end, we define a real symmetric tracefree tensor $P_{a b}=\chi_{A B} \bar{\chi}_{A^{\prime} B^{\prime}}$.

Lemma 2. There exists a vector $P^{a}$ such that

$$
\nabla_{(a} P_{b c)}=P_{(a} g_{b c)} .
$$

Proof. We first obtain the identity

$$
\frac{1}{2} \delta_{\left(a_{0}\right.}^{(a}{ }^{b c)} g_{\left.b_{0} c_{0}\right)}=\delta_{a_{0}}^{(a} \delta_{b_{0}}^{b} \delta_{c_{0}}^{c)}-\varepsilon_{A_{0}}{ }^{(A} \varepsilon_{B_{0}}{ }^{B} \varepsilon_{C_{0}}{ }^{C)} \varepsilon_{A_{0}^{\prime}}{ }^{\left(A^{\prime}\right.} \varepsilon_{B_{0}^{\prime}}{ }^{B^{\prime} \varepsilon_{C_{0}^{\prime}}{ }^{\left.C^{\prime}\right)} .}
$$

Denoting the left-hand side of (8) by $E_{a_{0} b_{0} c_{0}}^{a b c}$ and the right-hand side by $F_{a_{0} b_{0} c_{0}}^{a b c}$, we observe that each is idempotent:

that

$$
E_{a_{0} b_{0} c_{0}}^{a b c} E_{a_{1} b_{1} c_{1}}^{a_{0} b_{0} c_{0}}=E_{a_{1} b_{1} c_{1}}^{a b c} \quad F_{a_{0} b_{0} c_{0}}^{a b c} F_{a_{1} b_{1} c_{1}}^{a_{0} b_{0} c_{0}}=F_{a_{1} b_{1} c_{1}}^{a b c} ;
$$

$$
E_{a_{0} b_{0} c_{0}}^{a b c} F_{a_{1} b_{1} c_{1}}^{a_{0} b_{0} c_{0}}=E_{a_{1} b_{1} c_{1}}^{a b c}=F_{a_{0} b_{0} c_{0}}^{a b c} E_{a_{1} b_{1} c_{1}}^{a_{0} b_{0} c_{0}}
$$

and finally that the traces are equal:

$$
E_{a b c}^{a b c}=F_{a b c}^{a b c}=4
$$

(since $\delta_{\alpha}^{(\alpha} \delta_{\beta}^{\beta} \delta_{\gamma}^{\gamma)}=\frac{1}{6} n(n+1)(n+2)$ if $\delta_{\alpha}^{\beta}$ is an $n$-dimensional Kronecker delta). The required equality $E_{a_{0} b_{0} c_{0}}^{a b c}=F_{a_{0} b_{0} c_{0}}^{a b c}$ now follows. For, the above relations imply that the left range of $F_{a_{0} b_{0} c_{0}}^{a b c}$ contains the left range of $E_{a_{0} b_{0} c_{0}}^{a b c}$, but also that these ranges have the same dimension (namely 4), so they are equal. Similarly the right ranges are equal. An idempotent is uniquely determined once its left and right ranges are both fixed. Thus Eq. (8) is established.

Now we apply Eq. (8) by transvecting it on both sides with $\nabla_{a} P_{b c}$. Noting that $\nabla_{a} P_{b c}=\bar{\chi}_{B^{\prime} C^{\prime}} \nabla_{A^{\prime} A} \chi_{B C}+\chi_{B C} \nabla_{A A^{\prime}} \bar{\chi}_{B^{\prime} C^{\prime}}$ and using (5), we see that $P_{\left(a_{0}\right.} g_{\left.b_{0} c_{0}\right)}=\nabla_{\left(a_{0}\right.} P_{\left.b_{0} c_{0}\right)}$ where $P_{a}=\frac{1}{2} g^{b c} \nabla_{(a} P_{b c)}$, which establishes Lemma 2.

Lemma 3. The conformal Killing tensor $P_{b c}$ is irreducible provided the spacetime admits fewer than four independent Killing vectors.

Proof. Every type $\{22\}$ solution admits either a two or a four parameter group of isometries [16]. Since the geodesic equations admit four first integrals in the latter case, we restrict our attention to those solutions possessing only two independent Killing vectors. We wish to show that the tensor $P_{a b}$ is not expressible as a linear combination of the metric and the Killing tensors which are symmetric outer products of the two independent Killing vectors. The two Killing vectors span a 2-plane $T$ at each point and, as $T$ is non-null almost everywhere, we need only consider regions in which $T$ is timelike or spacelike. 
If $T$ is timelike in some region of the spacetime, then except perhaps on certain (at most) three-dimensional subsets, $T$ does not contain a repeated principal null vector. At some generic point $p$, let the real null vectors in $T$ be $\alpha^{A} \bar{\alpha}^{A^{\prime}}$ and $\beta^{A} \beta^{-A^{\prime}}$. Then, at $p$, the reducible Killing tensors are linear combinations of $\varepsilon_{A B} \varepsilon_{A^{\prime} B^{\prime}}, \alpha_{A} \alpha_{B} \bar{\alpha}_{A^{\prime}} \bar{\alpha}_{B^{\prime}}, \alpha_{(A} \beta_{B)} \bar{\alpha}_{\left(A^{\prime}\right.} \bar{\beta}_{\left.B^{\prime}\right)}$, and $\beta_{A} \beta_{B} \bar{\beta}_{A^{\prime}} \bar{\beta}_{B^{\prime}}$. These three expressions are annihilated when transvected with $\alpha^{A} \alpha^{B} \bar{\beta}^{A^{\prime}} \bar{\beta}^{B^{\prime}}$, but $\chi_{A B} \bar{\chi}_{A^{\prime} B^{\prime}}$ is not since none of $\alpha^{A} o_{A}, \alpha^{A} l_{A}, \beta^{A} o_{A}, \beta^{A} o_{A}$, or $\beta^{A} l_{A}$ vanishes. Thus, $\chi_{A B} \bar{\chi}_{A^{\prime} B^{\prime}}$ is irreducible when one of the Killing vectors is timelike.

If, on the other hand, $T$ is spacelike in some region, then $T$ will be spanned by complex linear combinations of a pair of complex null vectors $\alpha^{A} \bar{\beta}^{B^{\prime}}$ and $\beta^{A} \bar{\alpha}^{A^{\prime}}$. It is known in this case that, at a generic point, neither of the repeated principal null vectors $l^{a}$ or $n^{a}$ is orthogonal to both spacelike Killing vectors ${ }^{5}$. Therefore, none of the scalars $\alpha^{A} o_{A}$, $\alpha^{A} l_{A}, \beta^{A} o_{A}, \beta^{A} l_{A}$ vanishes, except perhaps on certain three dimensional subsets of the spacetime. The reducible Killing tensors in this case are linear combinations of $\alpha_{A} \alpha_{B} \bar{\beta}_{A^{\prime}} \bar{\beta}_{B^{\prime}}, \alpha_{(A} \beta_{B)} \bar{\alpha}_{\left(A^{\prime}\right.} \beta_{\left.B^{\prime}\right)}, \beta_{A} \beta_{B} \bar{\beta}_{A^{\prime}} \bar{\beta}_{B^{\prime}}$, and $\varepsilon_{A B} \varepsilon_{A^{\prime} B^{\prime}}$, and the fact that $\chi_{A B} \bar{\chi}_{A^{\prime} B^{\prime}}$ is irreducible then follows, as before, by transvection with $\alpha^{A} \alpha^{B} \bar{\alpha}^{A^{\prime}} \bar{\alpha}^{B^{\prime}}$ at a generic point.

This completes the proof of Theorem 1 .

A quantity such as $\chi_{B C}$, satisfying (5), could be called a Killing spinor and has some interest in its own right. Note that if

then

$$
\kappa^{A} \bar{\kappa}^{A^{\prime}} \nabla_{A A^{\prime}} \kappa_{B}=0
$$

$$
\kappa^{A} \bar{\kappa}^{A^{\prime}} \nabla_{A A^{\prime}}\left(\kappa^{B} \kappa^{C} \chi_{B C}\right)=0
$$

by virtue of Eq. (5). If Eq. (9) holds, then the null vectors $k^{a}=\kappa^{A} \bar{\kappa}^{A^{\prime}}$ are tangent to null geodesics $\gamma$ (since (9) implies $k^{c} \nabla_{c} k^{a}=0$ ). Eq. (10) tells us that the complex scalar $\chi=\kappa^{B} \kappa^{C} \chi_{B C}$ is constant along $\gamma$. The real scalar $|\chi|^{2}$ is the conformal quadratic first integral obtained from the conformal Killing tensor $P_{a b}$. But in addition, $\arg \chi$ is also constant along $\gamma$ and this gives us further information. We have seen that the constancy of $|\chi|^{2}$ helps us to locate the null geodesics in $M$. In an analogous way, the constancy of $\arg \chi$ enables us to determine the parallel propagation of "flag planes" (i.e., polarization planes) along $\gamma$. For, Eq. (9) states, in addition to the fact that the $k^{a}$ are tangent to null geodesics, that the flag plane (cf., $[9,11]$ ) of $\kappa^{A}$ is also parallelly propagated along $\gamma$. Knowledge of $\chi_{A B}$ defines for us this parallel propagation since (for example) if $\chi>0$ at one point of $\gamma$, then $\chi>0$ also at every other point of $\gamma$. This fixes the flag plane of $\kappa^{A}$ in relation to $\chi_{A B}$.

\footnotetext{
${ }^{5}$ We wish to thank W. Kinnersley for discussions on this point.
} 
Thus, knowledge of any (non-zero) $\chi_{A B}$ satisfying (5) supplies the information of the propagation of polarization of (say) a photon along any null geodesic $\gamma$.

The same would also hold for any "Killing spinor" with arbitrary (unequal) numbers of (symmetric) upper and lower indices:

with

$$
\nabla_{\left(B_{0}\right.}^{\left(A^{\prime} 0\right.} \chi_{\left.B_{1} \ldots B_{s}\right)}^{\left.A^{\prime}{ }_{1} \ldots A^{\prime} r\right)}=0
$$

$$
\chi=\kappa^{B_{1}} \ldots \kappa^{B_{s}} \bar{\kappa}_{A^{\prime}} \ldots \bar{\kappa}_{A_{r}^{\prime}} \chi_{B_{1} \ldots B_{s}^{\prime}}^{A_{1}^{\prime} \ldots B_{r}^{\prime}} .
$$

Eq. (11) is of interest also in other contexts. In flat spacetimes it represents in a conformally invariant way the general trace-free symmetric twistor $[13,14]$ of valence $\left(\begin{array}{l}r \\ s\end{array}\right)$.

Eq. (7) can be put in a form which is more suitable for the calculations involved in the proof of Theorem 2 as follows. Letting

$$
o^{A} \bar{o}^{A^{\prime}}=l^{a}, \quad l^{A} \bar{l}^{A^{\prime}}=n^{a}, \quad \text { and } \quad 6^{-2 / 3} \Psi_{2}^{-1 / 3} \bar{\Psi}_{2}^{-1 / 3}=\psi^{-1 / 3} \bar{\psi}^{-1 / 3}=\Sigma
$$

we have

$$
P_{b c}=\Sigma\left(l_{(b} n_{c)}-\frac{1}{4} g_{b c}\right),
$$

since $P_{b c}$ is tracefree and $l_{a} n^{a}=1$. Define $Q_{b c}=\Sigma l_{(b} n_{c}$. Then the equation $\nabla_{(a} P_{b c)}=P_{(a} g_{b c)}$ implies

$$
\nabla_{(a} Q_{b c)}=Q_{(a} g_{b c)}
$$

where $Q_{a}=P_{a}+\frac{1}{4} \nabla_{a} \Sigma$. Suppose now that there exists a scalar $Q$ such that $Q_{a}=\nabla_{a} Q$, and let $K_{b c}=Q_{b c}-Q g_{b c} ;$ then $\nabla_{(a} K_{b c)}=\nabla_{(a} Q_{b c)}$ $-Q_{(a} g_{b c)}=0$ and $K_{b c}$ is a quadratic Killing tensor.

Lemma 4. The Kerr solution [10] admits $K_{b c}$ as a quadratic Killing tensor.

Proof. The proof consists in finding the vector $Q^{a}$ given above and showing that $Q_{a}$ is a gradient. The Kerr metric has been expressed in a variety of coordinate systems $[10,15,3]$. For the purposes of the explicit calculation to follow, the most convenient of these is

$$
{ }_{\mathfrak{a} b}=\left(\begin{array}{lccl}
1-2 m r R^{-2} & -1 & 0 & 2 m r a^{2} R^{-2} \sin ^{2} \theta \\
-1 & 0 & 0 & a \sin ^{2} \theta \\
0 & 0 & -R^{2} & 0 \\
2 m r a^{2} R^{-2} \sin ^{2} \theta & a \sin ^{2} \theta & 0 & -\left(r^{2}+a^{2}+2 m r a^{2} R^{-2} \sin ^{2} \theta\right) \sin ^{2} \theta
\end{array}\right)
$$

where $\mathfrak{a}, \mathfrak{b}=0,1,2,3 ; x^{0}=u, x^{1}=r, x^{2}=\theta, x^{3}=\varphi$, and $R^{2}=r^{2}$ $+a^{2} \cos ^{2} \theta$. There are two Killing vectors with components, in these 
coordinates $\xi^{a}=(1,0,0,0)$ and $\eta^{a}=(0,0,0,1)$, corresponding to the fact that $g_{\mathfrak{a} b}$ is independent of $u$ and $\varphi$. The contravariant components of $g_{a b}$ are

$$
g^{\mathfrak{a} \mathfrak{b}}=\left(\begin{array}{lccl}
-a^{2} R^{-2} \sin ^{2} \theta & -\left(r^{2}+a^{2}\right) R^{-2} & 0 & -a R^{-2} \\
-\left(r^{2}+a^{2}\right) R^{-2} & \left(2 m r-r^{2}-a^{2}\right) R^{-2} & 0 & -a R^{-2} \\
0 & 0 & -R^{-2} & 0 \\
-a R^{-2} & -a R^{-2} & 0 & -R^{-2} \sin ^{-2} \theta
\end{array}\right) .
$$

The repeated principal null vectors $l^{a}$ and $n^{a}$ to which the above coordinates $\{u, r, \theta, \varphi\}$ are adapted, have components

$$
\begin{aligned}
l^{a} & =(0,-1,0,0), \\
n^{a} & =R^{-2}\left(r^{2}+a^{2}, \frac{1}{2}\left(r^{2}+a^{2}-2 a m r\right), 0, a\right),
\end{aligned}
$$

and satisfy $l^{a} \nabla_{\mathrm{a}} l^{\mathfrak{c}}=0, l_{\mathrm{a}} n^{\mathrm{a}}=1$. The latter normalization precludes affine parametrization of $n^{a}$. The complex convergence $\varrho$ of the null geodesic congruence defined by $l^{a}$ is given [12] by $\varrho=-(r-i a \cos \theta)^{-1}$, and the only nonvanishing dyad component of the Weyl spinor is $\Psi_{2}=-m \varrho^{3}$ where $m$ is the mass parameter. Therefore

$$
\Psi_{2}^{-1 / 3} \bar{\Psi}_{2}^{-1 / 3}=m^{-2 / 3}\left(r^{2}+a^{2} \cos ^{2} \theta\right)=m^{-2 / 3} R^{2} .
$$

The scalar $\Sigma$ of Eq. (12) is given by $\Sigma=(6 m)^{-2 / 3} R^{2}$. Absorbing the constant factor into $Q_{b c}$ we put $Q_{\mathrm{bc}}=\frac{1}{2} R^{2} l_{(\mathrm{b}} n_{\mathrm{c})}$ so that

$$
Q^{b c}=\left(\begin{array}{llll}
0 & r^{2}+a^{2} & 0 & 0 \\
r^{2}+a^{2} & r^{2}+a^{2}-2 a m r & 0 & a \\
0 & 0 & 0 & 0 \\
0 & a & 0 & 0
\end{array}\right)
$$

The calculation of the components of the vector $Q^{a}$ satisfying $\nabla^{(a} Q^{b c)}=Q^{(a} g^{b c)}$ is greatly simplified by noting ${ }^{6}$ that

$$
\nabla^{(a} Q^{b c)}=g^{e(a} \partial_{e} Q^{b c)}-Q^{e(a} \partial_{e} g^{b c)}
$$

in any coordinate system, where $\partial_{\mathrm{e}}$ denotes the ordinary partial derivative $\partial / \partial x^{\mathrm{e}}$.

The vector $Q^{a}$ is then defined by

$$
Q^{(\mathfrak{a}} g^{b \mathfrak{c})}=g^{\mathrm{e}(\mathfrak{a}} \partial_{\mathrm{e}} Q^{\mathrm{bc})}-Q^{\mathrm{e}(\mathfrak{a}} \partial_{\mathrm{e}} g^{\mathrm{bc})} .
$$

The calculation is further simplified by noting that $Q^{2 a}=0$ so that the $\partial_{2} g^{b c}=(\partial / \partial \theta) g^{\mathfrak{b c}}$ are not required, and also that $\partial_{2} Q^{\mathbf{b c}}=0$. We find

$$
Q^{a}=2 r R^{-2}\left(r^{2}+a^{2}, r^{2}+a^{2}-2 m r, 0, a\right)
$$

\footnotetext{
${ }^{6}$ We wish to thank R. Geroch for pointing out this fact to us.
} 
so that $Q_{\mathfrak{a}}=g_{\mathfrak{a} \mathfrak{c}} Q^{\mathfrak{c}}=(0,-2 r, 0,0)=\nabla_{\mathfrak{a}}\left(-r^{2}\right)$. We conclude that the components

$$
K^{\mathfrak{a} \mathfrak{b}}=Q^{\mathfrak{a} \mathfrak{b}}+r^{2} g^{\mathfrak{a} \mathfrak{b}}
$$

define a quadratic Killing tensor for the Kerr solution. Explicitly,

$$
K^{\mathrm{ab}}=\left(\begin{array}{llll}
-r^{2} a^{2} R^{-2} \sin ^{2} \theta & \left(r^{2}+a^{2}\right) R^{-2} a^{2} \cos ^{2} \theta & 0 & -a r^{2} R^{-2} \\
\left(r^{2}+a^{2}\right) & \left(r^{2}+a^{2}-2 m r\right) & 0 & a^{3} R^{-2} \cos ^{2} \theta \\
\cdot R^{-2} a^{2} \cos ^{2} \theta & \cdot R^{-2} a^{2} \cos ^{2} \theta & & \\
0 & 0 & -r^{2} R^{-2} & 0 \\
-a r^{2} R^{-2} & a^{3} R^{-2} \cos ^{2} \theta & 0 & -r^{2} R^{-2} \sin ^{-2} \theta
\end{array}\right)
$$

In order to complete the proof of Theorem 2 we note that the contravariant components of the metric of the charged Kerr solution with cosmological constant are obtained from those of the metric of the Kerr solution by replacing $2 m r-r^{2}-a^{2}$ in $g^{11}$ of Eq. (15) by $2 m r-r^{2}-a^{2}$ $+e^{2}+\frac{1}{3} \Lambda r^{4}$, where $e$ is the charge parameter and $\Lambda$ the cosmological constant. Replacing $K^{11}$ in Eq. (18) by $R^{-2}\left(r^{2}+a^{2}-2 m r-e^{2}-\frac{1}{3} \Lambda r^{4}\right)$ - $a^{2} \cos ^{2} \theta$, a straightforward calculation shows that $\nabla^{(\mathfrak{a}} K^{\mathrm{bc})}=0$ in the more general case also.

Acknowledgements. We wish to thank P. Havas and R. Geroch for stimulating discussions during the course of this work.

\section{References}

1. Carter, B.: Commun. Math. Phys. 10, 280 (1968).

2. Newman, E. T., Couch, E., Chinnapared, R., Exton, A., Prakash, A., Torrence, R.: J. Math. Phys. 6, 918 (1965).

3. Carter, B.: Phys. Rev. 174, 1559 (1968).

4. Geroch, R.: Ann. Phys. 48, 526 (1968).

5. Penrose, R.: Proc. Roy. Soc. A 284, 159 (1965).

6. Geroch, R.: J. Math. Phys. 9, 450 (1968).

7. Eisenhart, L. P.: Riemannian geometry, p. 128. Princeton, N. J.: Princeton University Press 1966.

8. Schouten, J. A.: Ricci calculus, 2nd edition, p. 293. Berlin, Göttingen, Heidelberg: Springer 1954.

9. Pirani, F. A. E.: Introduction to gravitational radiation theory, Lectures on general relativity, Brandeis Summer Institute (1964), Vol. I, S. Deser and K. Ford editors. Englewood Cliffs, N. J.: Prentice-Hall, Inc. 1965.

10. Kerr, R. P.: Phys. Rev. Letters 11, 237 (1963).

11. Penrose, R.: Structure of space-time. Battelle rencontres. C. de Witt and J. A. Wheeler, Eds. New York: W. A. Benjamin Inc. 1968.

12. Newman, E. T., Penrose, R.: J. Math. Phys. 3, 566 (1962). 
13. Penrose, R.: J. Math. Phys. 8, 345 (1967).

14. - Int. J. Theor. Phys. 1, 61 (1968).

15. Boyer, R. H., Lindquist, R. W.: J. Math. Phys. 8, 265 (1967).

16. Kinnersley, W.: J. Math. Phys. 10, 1195 (1969).

\author{
M. Walker, \\ R. Penrose \\ Department of Physics \\ University of Texas at Austin \\ Austin, Texas 78712, USA
}

